\title{
Study of Aerobic Bacterial Etiology and Their Antibiogram from Pus Samples in a Tertiary Care Hospital
}

\author{
T. Kanakadurgamba ${ }^{1}$, Rama Lakshmi Koripella ${ }^{2}$, B. Gowtham ${ }^{3}$, \\ Appa Rao Peddapalli ${ }^{4}$
}

${ }^{1,2}$ Assistant Professor, ${ }^{3}$ Post-Graduate, ${ }^{4}$ Professor and HOD, Department of Microbiology, Andhra Medical College, Visakhapatnam, Andhra Pradesh, India.

Corresponding Author: Rama Lakshmi Koripella

\begin{abstract}
Introduction: The spread of multidrug resistant bacteria has added a new angel to the problem of wound infections which are a major cause of morbidity. To avoid difficulty in treatment of such infections it is mandatory for every pus sample to undergo Culture and sensitivity.

Aim: To isolate and study the antibiogram of the aerobic bacteria from pus samples.

Materials and Methods: A total of 426 pus samples received from various wards in the Microbiology department. Andhra Medical College, Visakhapatnam were processed as per standard guidelines in the laboratory. All the aerobic bacterial isolates obtained were kept for antibiogram by Kirby Bauer's disc diffusion method as per CLSI Guidelines.
\end{abstract}

Results: Out of 426 samples 394 (92.4\%) were culture positive. Among culture positive samples $90.6 \%$ were pure cultures and $9.4 \%$ were mixed isolates. Gram negative bacilli were $72.8 \%$ of the total isolates and $27.2 \%$ were Gram positive cocci. Escherichia coli and Klebsiella pneumonia were the predominant isolates $(30.3 \%$ and $28.9 \%)$ followed by Pseudomonas aeruginosa (24\%), Proteus species (9.7\%) and Acinetobacter species (6.9\%). Among Gram positive cocci Staphylococcus aureus (62.6\%), Enterococci (20.5\%) and Coagulase Negative Staphylococcus (11.2\%) and Streptococci (3.7\%). GNB were more sensitive to Colistin (92\%), Amikacin (86\%), Imipenem and Meropenem (86\% and $84.4 \%$ ), Piperacillin Tazobactam (84\%), Gentamicin (78\%), Cefaperazone Sulbactam $(76.2 \%)$ and Ciprofloxacin (56\%). Gram positive cocci were sensitive to Linezolid and Teicoplanin (98\% each), Vancomycin (96.2\%),

Clindamycin (84\%), Gentamicin (79\%), Azithromycin (76.4\%), Ciprofloxacin (66\%) and Amoxiclav (54\%) out of $62.6 \%$ Staphylococcus aureus isolates $31.4 \%$ were MRSA and out of $72.8 \%$ of Gram negative bacilli $38.2 \%$ were ESBLS. Conclusion: As multidrug resistant bacterial strains are emerging in various infectious and pyogenic wound infection is most important cause of morbidity, it is important for a clinician to send all pus samples for microbiological analysis and their antibiogram before putting cases on

Key words: Pus samples, Multidrug resistance, Gram negative bacilli, Gram positive cocci, Antimicrobial sensitivity.

\section{INTRODUCTION}

Antibiotic resistance among bacteria is becoming more and more serious problem throughout the world. It is said that evolution of bacteria towards resistant to antimicrobial drugs, including multidrug resistance, is unavoidable because it represents a particular aspect of the general evolution of bacteria that is un-stoppable ${ }^{1}$.

Pyogenic wound infection is major cause of morbidity. The pathogens isolated from infections differ depending on the underlying problem, location and type of surgical procedure. Most common organisms encountered are staphylococcus aureus, Klebsiella species, Escherichia coli, 
Pseudomonas species, Proteus species, Enterococci species ${ }^{2}$, Enterobacter, Candida and Acinetobacter species ${ }^{3}$.

Wound infection can be caused by variety of organisms like Bacteria, Virus, Fungi and Protozoa and may co-exist as polymicrobial communities especially in wound margins and in chronic wounds ${ }^{4}$. In many cases there is a mixed infection with more than one bacterial species ${ }^{5}$.

Staphylococcus aureus is most important pathogens in skin as well as soft tissue infections. Methicillin Resistant Staphylococcus aureus (MRSA) is prevalent in majority of the countries whenever it is sought for. MRSA is one of the important pathogens in hospitals acquired infectious ${ }^{4,6}$.

The spread of multidrug resistant bacterial pathogens has added a new angel to the problem of wound infections. This is particularly worse in resource poor countries where sale of antibiotics is under poor control ${ }^{7}$.

A number of studies have been carried out in the west to monitor antimicrobial resistance at national level. The Academic and educational value of these studies is particularly useful for Microbiologists and infectious disease clinicians. The data collected from these studies are useful in improving antimicrobial use in those communities ${ }^{8-11}$.

The present study is an attempt to know the current status of antibiotic sensitivity pattern of aerobic bacterial isolates in a Tertiary Care hospital of Visakahapatnam, Andhra Pardesh.

\section{MATERIALS AND METHODS}

A total of 426 pus samples received in the department of Microbiology, Andhra Medical College, Visakhapatnam in 2019 were processed as per the standard guidelines in the laboratory. The samples were collected from various wards in King George Hospital, Visakhapatnam. Gram's staining was done for all the samples and inoculated on Sheep blood agar and Macconkeys agar and incubated at $37^{*} \mathrm{C}$ for 24 hours. Organisms were isolated and identified by standard biochemical reactions and antibiotic sensitivity was done on Muller Hinton agar by Kirby Bauer's disc diffusion method as per CLSI Guidelines.

\section{RESULTS}

Out of 426 pus samples processed, $92.4 \%$ (394) were culture positive and 7.6\% (32) samples were culture sterile for aerobic growth.

Among 394 culture positive samples majority were from the male patients $58.6 \%$ (231) and 41.4\% (163) were from females. Maximum samples were positive for culture in the age group of 21 to 30 years $30.2 \%$ (119) followed by 51 to 60 years $21.8 \%$ (86).

Out of 394 cultures positive samples $90.6 \%$ were pure cultures and $9.4 \%$ were mixed isolates. Gram negative bacilli were $72.8 \%$ of the total isolates and $27.2 \%$ were Gram positive cocci. Escherichia coli and Klebsiella Pneumoniae were the predominant isolates $(30.3 \%$ and $28.9 \%)$ followed by Pseudomonas aeruginosa (24\%), Proteus species (9.7\%) and Acinetobacter species (6.9\%). Among Gram positive cocci Staphylococcus aureus (62.6\%), Enterococci (20.5\%) and Coagulase Negative Staphylococcus (11.2\%) and Streptococci (3.7\%). GNB were more sensitive to Colistin (92\%), Amikacin (86\%), Imipenem and Meropenem (86\% and 84.4\%), Piperacillin Tazobactam (84\%), Gentamicin (78\%), Cefaperazone Sulbactam (76.2\%) and Ciprofloxacin (56\%). Gram positive cocci were sensitive to Linezolid and Teicoplanin (98\% each), Vancomycin (96.2\%), Clindamycin (84\%), Gentamicin (79\%), Azithromycin (76.4\%), Ciprofloxacin (66\%) and Amoxiclav (54\%) out of $62.6 \%$ Staphylococcus aureus isolates $31.4 \%$ were MRSA and out of $72.8 \%$ of Gram negative bacilli $38.2 \%$ were ESBLS.

\section{DISCUSSION}

The microbial pathogens, as well as their antibiotic sensitivity pathogens may change from time to time and place to place. 
The discovery of antibiotics revolutionized the management of infectious diseases. However, the overuse and misuse of antibiotics is leading to the emergence of resistance to these lifesaving drugs. Hospitals Antibiograms are commonly used to help and guide empiric antimicrobial treatment and are an important component of detecting and monitoring trends in antimicrobial resistance ${ }^{12}$.

The microbiology laboratory plays a central role in the decision to choose a particular antimicrobial agent over others. Once the microbial species causing the disease is identified, a rational choice of the class of antibiotics likely to work on the patient can be made ${ }^{13}$.

In the present study, culture positivity was observed in $92.4 \%$ of pus samples which correlates with Vijeta Sharma etal ${ }^{14}$ who reported $93 \%$ of culture positivity, Swati Duggal etal ${ }^{15}$ (93.27\%), Nirmala Grace etal ${ }^{16}$ (90.8\%), whereas Asmabegaum Birdar etal ${ }^{17}$ and Hanumanthappa etal ${ }^{18}$ reported lower incidence of $66 \%$ and $56 \%$ of culture positivity in their studies.

Among culture positive samples 90.6\% were Pure Cultures and 94\% were Mixed Cultures which correlate with Asmabeguam Birdar etal ${ }^{17}$ who reported $92.89 \%$ and $7.1 \%$, Prajuli etal ${ }^{4}(92 \%$ and $8 \%)$, Nirmala etal ${ }^{16}(88.2 \%$ and $11.8 \%)$ and Mohanth etal ${ }^{19}$ who reported $(85.8 \%$ and $14.2 \%)$.

In the present study Gram negative bacilli isolated were $72.8 \%$ out of 394 cultures positive samples and Grampositive cocci were $27.2 \%$ which correlates with Nirmala etal $^{16} \quad(73.7 \%$ and $26.3 \%)$, Asmabegaum Birdar etal ${ }^{17}$, Jain etal ${ }^{7}$, Ghosh etal ${ }^{20}$ and Hanumanthappa etal ${ }^{18}$ where as Prajuli etal ${ }^{4}$ in their study isolated more of Gram positive bacteria compared to Gram negative bacilli.

Among Gram negative bacilli, Escherichia coli and Klebsiella Pneumoniae were the predominant isolates followed by Pseudomonas species which correlates with Iffat Javeed etal ${ }^{21}$, whereas Vijetha Sharma etal $^{14}$, Rajeshwar Rao etal ${ }^{22}$ who reported Klebsiella as predominant isolate and Farzana $\mathrm{R}$ etal ${ }^{23}$, Ehmer Abraham etal ${ }^{24}$ and Soumya Kaup etal ${ }^{25}$ reported Pseudomonas as the predominant isolate in their studies.

Staphylococcus was the predominant isolate among Gram positive cocci in the present study which correlates with Asmabegaum Birdar etal ${ }^{17}$, Swathi Duggal etal $^{15}$, Nirmala etal ${ }^{16}$, whereas Vijetha Sharma etal ${ }^{14}$ reported very lower incidence.

The antibiotic susceptibility pattern of Gram negative bacilli and Gram positive cocci in the present study correlates with Nirmala etal ${ }^{16}$, Vijetha Sharma etal ${ }^{14}$, Soumya Kaup etal ${ }^{25}$, Rajeshwar etal ${ }^{22}$, Balan etal $^{26}$, Asmabegaum etal ${ }^{17}$ and Swathi Duggal etal ${ }^{15}$.

In the present study MRSA was isolated in $31.4 \%$ of Staphylococcus aureus which correlates with Nirmala etal ${ }^{16}$ who reported $27 \%$, Swathi Duggal etal ${ }^{15}$ reported $35.9 \%$ and Asmabegaum Birdar etal ${ }^{17}$ (39.47\%). Out of $72.8 \%$ of Gram negative bacilli $38.2 \%$ were ESBLs in the present study. Raghunath D etal ${ }^{27}$ reported in 2008 that coliforms have changed their susceptibility patterns extensively. According to them, $\beta$ Lactam resistance is widespread among coliform bacteria due to vertical as well as horizontally acquired resistance factors. Researchers from Lahore have reported that resistance to $\beta$ Lactam drugs amongst Escherichia coli and Klebsiella is alarming with resistance ranging from $35.5 \%$ to $43.82 \%{ }^{8,29}$.

\section{CONCLUSION}

Knowledge of the most common causative agents of infection and their antimicrobial susceptibility pattern is very essential for the judicious administration of empirical therapy before the culture results are available. Antimicrobial susceptibility of microorganisms varies from time to time and from place to place. Pyogenic wound infection is most important cause of morbidity and as multidrug resistant bacterial strains are emerging most commonly, it is important for a clinician to 
T. Kanakadurgamba et.al. Study of aerobic bacterial etiology and their antibiogram from pus samples in a tertiary care hospital.

send all Pus samples for microbiological analysis and their antibiogram before putting cases on antibiotics.

\section{Acknowledgement: None}

\section{Conflict of Interest: None}

Source of Funding: None

\section{Ethical Approval: Approved}

\section{REFERENCES}

1. Courvalin P .Antimicrobial Drug Resistance: "Prediction Is Very Difficult, especially about the Future". Emerg Infect Dis. 2005; 11: 1503-06.

2. Krige JEJ., and Beckingham JI.2001. Liver abscesses and hydatid disease. BMJ 23;322(7285):537.

3. Tayfour MA, Al-Ghamdi SM and Alahamdi AS. Surgical wound infections in King Fahed Hospital at Al-baha Saudi Med. J. 2005;26(8):1305-07.

4. Parajuli P, Basnyat SR, Shrestha R, Shah PK, Gurung P. Identification and Antibiotic Susceptibility Pattern of Aerobic Bacterial Wound Isolates In Scheer Memorial Hospital. JSM Microbiology 2014;2(2):1011.

5. Collee JG, Fraser AG, Marmion BP, Simmons A, editors. Mackie and McCartney - Practical Medical Microbiology. 14th ed., Ch. 4. New Delhi: Elsevier 2007;53-94.

6. Mumtaz S, Akhtar $\mathrm{N}$ and Hayat A. Antibiogram of aerobic pyogenic isolates from wounds and abscesses of patients at Rawalpindi. Pak. J. Med. Res. 2002;41:8993.

7. Jain V, Ramnani VK and Kaore N. Antimicrobial susceptibility pattern amongst aerobic bacteriological isolates in infected wounds of patients attending tertiary carehospital in Central India. Int. J. Curr. Microbiol. App. Sci. 2015;4(5):711-719.

8. Anwar M.S, Chaudhary I, Ahmad I, Bhatti K.UR., Jaffery G, Tayyab M, Bokhari S.R. Frequency of extended spectrum beta lactamase producing Klebsiella pnumoniae and Escherichia coli isolates. Biomedica 2007; 23: 34-38.

9. Lakshmi V. Need for national/regional guidelines and policies in India to combat antibiotic resistance. Indian $\mathbf{J}$ Med Microbiol 2008; 26: 105-7.

10. Joshi A, Iyer V, Balasubramaniam U, Kagal A, Bharadwaj R. Comparison of efficacy of three comercially available antibiotic discs. Indian J Med Microbiol 2008; 26: 160-2.

11. Latif S, Anwar M S, Ahmad I. Bacterial pathogens responsible for blood stream infection (BSI) and pattern of drug resistance in a tertiary care hospital of Lahore. Biomedica 2009; 25: 101-105.

12. The utility of hospital antibiograms as tool for guiding empiric therapy and tracking resistance: Insight from the society of infectious diseases pharmacists. Pharmacotherapy 2007; 27: 1306-12.

13. Henry Chambers F. and Goodman \& Gillmans. The Pharmacological basis of Therapeutics, General principles of antimicrobial therapy. 12th edition, New York: McGraw-Hill; 2010: 1369

14. Vijeta Sharma, 2Geeta Parihar, 3Vijaylaxmi Sharma, 4Harshita Sharma. A Study of Various Isolates from Pus Sample with Their Antibiogram from Jln Hospital, Ajmer . DOI: 10.9790/0853-141066468

15. Swati Duggal1, P K Khatri2, R S Parihar3 , Rajat Arora4. Antibiogram of Various Bacterial Isolates from Pus Samples in a Tertiary Care Centre in Rajasthan. Volume 4 Issue 5, May 2015. Paper ID: SUB154568

16. B. Nirmala Grace, K. Ravi Kiran, B. Venkata Rao. Study of Aerobic Bacterial Isolates and Their Antibiogram from Pus Sample in Government General Hospital, Guntur. International Journal of Research and Review (ijrrjournal.com) Vol.7; Issue: 7; July 2020

17. Biradar A, Farooqui F, Prakash R, Khaqri SY, Itagi I. Aerobic bacteriological profile with antibiogram of pus isolates. Indian $\mathbf{J}$ Microbiol Res 2016;3(3):245-249

18. Hanumanthappa P, Vishalakshi B and Krishna S.A Study on aerobic Bacteriological profile and Drug sensitivity pattern of Pus samples in a tertiary care hospital. Int. J. Curr. Microbiol. App. Sci. 2016;5(1):95-102.

19. Mohanty S, Kapil A, Dhawan B, Das BK. Bacteriological and antimicrobial susceptibility profile of soft tissue infections from Northern India. Indian J Med Sci. 2004;58:10-15

20. Ghosh A, Karmakar PS, Pal J, Chakraborty N, Debnath NB, Mukherjee JD. Bacterial 
T. Kanakadurgamba et.al. Study of aerobic bacterial etiology and their antibiogram from pus samples in a tertiary care hospital.

incidence and antibiotic sensitivity pattern in moderate and severe infections in hospitalized patients. J Indian Med Assoc. 2009;107(1):21-24.

21. Iffat javeed, 1 rubeena hafeez1 and $\mathrm{m}$. saeed anwar2 .antibiotic susceptibility pattern of bacterial isolates from patients admitted to a tertiary care hospital in lahore . biomedica vol. 27 (jan. - jun. 2011)

22. S. Rajeshwar Rao, L. Jaya Lakshmi1, S.Pavani, Vijendra Kawle and S. Jaya Prakash. Bacteriological Profile, Antibiogram of Burn wound Isolates and Detection of MRSA and ESBL Production at Tertiary Care Hospital, Hyderabad. World Journal of Pharmacy and Pharmaceuticals Sciences. 2014; 3:16911698.

23. Farzana R, Shamsuzzaman SM, Mamun KZ, and Shears P. Antimicrobial susceptibility pattern of extended spectrum betalactamase producing gram-negative bacteria isolated from wound and urine in a tertiary care hospital, Dhaka City, Bangladesh. Southeast Asian J Trop Med Public Health. 2013; 44(1) : 96-103.

24. Ehmer Al-Ibran, Sidra Meraj, Mahwish Nasim, Maria Khan2 and Masood Hussain Rao. Pattern of Microorganisms Isolated from Flame Burn Wounds, and Their Trends of Susceptibility to Antibiotic
During Past Three Years. Journal of the Dow University of Health Sciences Karachi. 2013; 7: 49-53.

25. Soumya Kaup and Jaya Sankarankutty. Prevalence and antimicrobial susceptibility patterns of bacteria isolated from skin and wound infections. Journal of Microbiology and Biotechnology Research.2014; 4(2): 3945.

26. Balan K, Sujitha K, Vijayalakshmi TS. Antibiotic susceptibility pattern of gram negative clinical Isolates in a Teaching Tertiary Care hospital. Scholars Journal of Applied Medical Sciences 2013; 1(2): 7679.

27. Raghunath D. Emerging antibiotic resistance in bacteria with special reference to India J Biosci 2008; 33: 593-603.

28. Hafeez R, Aslam M, Mir F, Tahir M, Javeed I, Ajmal M. Frequency of extended spectrum beta lactamase producing gram negative bacilli among clinical isolates. Biomedica 2009; 25: 112-5

How to cite this article: T. Kanakadurgamba, Rama Lakshmi Koripella, B. Gowtham et.al. Study of aerobic bacterial etiology and their antibiogram from pus samples in a tertiary care hospital. International Journal of Research and Review. 2021; 8(4): 358-362. $\quad$ DOI: https://doi.org/10.52403/ijrr.20210442 